\title{
Spectral tau algorithm for solving a class of fractional optimal control problems via Jacobi polynomials
}

\author{
Youssri H. Youssri* and Waleed M. Abd-Elhameed \\ Department of Mathematics, Faculty of Science, Cairo University, Giza, Egypt \\ youssri@sci.cu.edu.eg,waleed@sci.cu.edu.eg
}

\section{ARTICLE INFO}

\section{Article History:}

Received 21 January 2017

Accepted 06 March 2018

Available 11 April 2018

Keywords:

Jacobi polynomials

Tau method

Newton's iterative method

Optimal control problems

System of fractional differential

equations

AMS Classification 2010:

$65 M^{7} 70,65 N 35,35 C 10,42 C 10$

\begin{abstract}
This paper is dedicated to presenting an efficient numerical algorithm for solving a class of fractional optimal control problems (FOCPs). The basic idea behind the suggested algorithm is based on transforming the FOCP under investigation into a coupled system of fractional-order differential equations whose solutions can be expanded in terms of the Jacobi basis. With the aid of the spectral-tau method, the problem can be reduced into a system of algebraic equations which can be solved via any suitable solver. Some illustrative examples and comparisons are presented aiming to demonstrate the accuracy, applicability and efficiency of the proposed algorithm.
\end{abstract}

\section{Introduction}

Fractional calculus is of course a very important branch of mathematical analysis. This branch of calculus is interested in generalizing the derivatives and integrals of integer order to include derivatives and integrals of an arbitrary order (real or complex). A large number of authors investigate fractional derivatives from both theoretical and practical points of view (see, for example, [1-4]). It is well-known that many physical phenomena in acoustics, damping laws, electroanalytical chemistry, neuron modeling, diffusion processing and material sciences (see for example, [5-7]) are described by fractional differential equations. Various algorithms are developed for handling different kinds of fractional differential equations. Some of these methods are, Adomian decomposition method [7, 8, variational iteration method [9] and fractional differential transform method [10,11] and ultraspherical wavelets method [12.
Optimal control problems arise in various applied sciences such as mechanics, aerospace engineering, and economics. The exact solutions for most of these problems are not easy to implement, so it is natural to try to derive numerical solutions for such problems. The general definition of an optimal control problem requires the minimization of a criterion function of the states and control inputs of the system over a set of admissible control functions. This system is governed by constrained dynamics and control variables. Additional constraints such as final time constraints can be considered, see for example [13, 14].

Spectral and pseudospectral methods have become increasingly popular as higher order methods for the solution of various differential equations represent the solution of a certain problem in a basis set of orthogonal functions. The main advantage of these methods is that they can provide exponential convergence of the solutions. These methods are employed for solving many problems appear in various branches of science such as

*Corresponding Author 
physics, chemistry and fluid mechanics. One can consult the monographs by Shen et al. [15], the books by Kopriva [16], and Shizgal [17] for some of these applications. There are three popular techniques for spectral methods, they are Galerkin, tau and collocation methods. Every method has its importance. The Galerkin approach requires to select suitable basis functions satisfying the boundary conditions and then enforcing the residual to be orthogonal with the basis functions. This method has been applied in a variety of papers. It has has been successfully applied to linear problems, see for example [18]19]. The collocation method is a suitable approach for treating nonlinear problems, see for example [20 23]. The tau method is a particular class of Petrov-Galerkin method. This method is often used for problems contain complicated boundary conditions.

Orthogonal polynomials in general and Jacobi polynomials in particular have distinguished parts in applied mathematical analysis. It is wellknown that the class of the Jacobi polynomials includes six subclasses of orthogonal polynomials, namely, Legendre, ultraspherical and the four kinds of Chebyshev polynomials. The Jacobi polynomials and their special polynomials are extensively used along with spectral methods for solving ordinary and fractional differential equations, see for instance [24 29].

This paper introduces a general numerical algorithm for a class of FOCPs. A FOCP is an optimal control problem in which the criterion and/or the differential equations governing the dynamics of the system contain at least one fractional derivative operator.

FOCP can be described by different definitions of fractional derivatives. The most commonly used types of fractional derivatives are RiemannLiouville and Caputo fractional derivatives. General necessary conditions of optimality have been developed for FOCPs. For instance, in [9, 11, the author has achieved Hamiltonian formulas for FOCPs with Riemann-Liouville fractional derivative, while the author in [30] has achieved some formulas with Caputo fractional derivative. Hamiltonian system of equations provides necessary conditions of optimization. The optimal solution of the FOCP should satisfy the system [31.

The main aim of this article is twofold:

- Reformulating fractional optimal control problem to a coupled system of fractionalorder differential equations.

- Analyzing efficient spectral-tau algorithm for handling the resulting system of fractional-order differential equations via Jacobi polynomials.

The rest of the paper is arranged as follows. The next section is devoted to presenting mathematical preliminaries containing some basic definitions in the fractional calculus theory which are required for establishing our results. Also, some relevant properties of Jacobi polynomials and their shifted ones are presented. In Section 3, we present an efficient spectral tau algorithm for solving FOCPs. In Section 4, we give some numerical examples to ensure the efficiency, simplicity and applicability of the suggested algorithm. Finally, Section 5 is devoted to presenting concluding remarks.

\section{Preliminaries}

This section is devoted to presenting some basic definitions and properties in fractional calculus. In addition, some relevant properties of Jacobi polynomials and their shifted ones are presented.

\subsection{Some definitions and properties of fractional calculus}

Definition 1. The Riemann-Liouville fractional integral operator $I^{\alpha}$ of order $\alpha$ on the usual Lebesgue space $L_{1}[0,1]$ is defined as

$I^{\alpha} f(t)= \begin{cases}\frac{1}{\Gamma(\alpha)} \int_{0}^{t}(t-\tau)^{\alpha-1} f(\tau) d \tau, & \alpha>0, \\ f(t), & \alpha=0 .\end{cases}$

The operator $I^{\alpha}$ has the following properties:

$$
\begin{aligned}
& \text { (i) } I^{\alpha} I^{\beta}=I^{\alpha+\beta}, \\
& \left(\text { ii } I^{\alpha} I^{\beta}=I^{\beta} I^{\alpha},\right. \\
& (i i i) I^{\alpha}(t-a)^{\nu}=\frac{\Gamma(\nu+1)}{\Gamma(\nu+\alpha+1)}(t-a)^{\nu+\alpha},
\end{aligned}
$$

where $f \in L_{1}[0,1], \alpha, \beta \geqslant 0$, and $\nu>-1$.

Definition 2. The Riemann-Liouville fractional derivative of order $\alpha>0$ is defined by

$\left(D^{\alpha} f\right)(t)=\left(\frac{d}{d t}\right)^{n}\left(I^{n-\alpha} f\right)(t), n-1 \leqslant \alpha<n, n \in \mathbb{N}$.

Definition 3. The Caputo fractional left and right differential operators are defined respectively as

$\left({ }_{0} D_{t}^{\alpha} f\right)(t)=\frac{1}{\Gamma(n-\alpha)} \int_{0}^{t}(t-\tau)^{n-\alpha-1} f^{(n)}(\tau) d \tau$, 
$\left({ }_{t} D_{1}^{\alpha} f\right)(t)=\frac{1}{\Gamma(n-\alpha)} \int_{t}^{1}(\tau-t)^{n-\alpha-1} f^{(n)}(\tau) d \tau$

where $n-1 \leqslant \alpha<n, n \in \mathbb{N}$.

The Caputo fractional differential operator satisfies the following basic formula, for $n-1 \leqslant \alpha<n$,

$$
\left(I^{\alpha} D^{\alpha} f\right)(t)=f(t)-\sum_{k=0}^{n-1} \frac{f^{(k)}\left(0^{+}\right)}{k !}(t-a)^{k}, t>0 .
$$

For more details on the mathematical properties of fractional derivatives and integrals, see for example, [3].

\subsection{Classical Jacobi polynomials}

For $\gamma, \delta \in \mathbb{R}, \gamma, \delta>-1$, and a nonnegative integer $n$, we denote by $P_{n}^{(\gamma, \delta)}(x)$ the Jacobi polynomial, which comprises all the polynomial solutions to singular Sturm-Liouville problems on $(-1,1)$. $P_{n}^{(\gamma, \delta)}(x)$ has the following Gauss hypergeometric representation:

$P_{n}^{(\gamma, \delta)}(x)=\frac{\Gamma(n+\gamma+1)}{n ! \Gamma(\gamma+1)}{ }_{2} F_{1}\left(-n, n+\gamma+1 ; \gamma+1 ; \frac{1-x}{2}\right)$

The Jacobi polynomials are orthogonal on $[-1,1]$ with the wight function $w^{(\gamma, \delta)}(x)=(1-x)^{\gamma}(1+$ $x)^{\delta}$, in the sense that

$\int_{-1}^{1}(1-x)^{\gamma}(1+x)^{\delta} P_{m}^{(\gamma, \delta)}(x) P_{n}^{(\gamma, \delta)}(x) d x=h_{n}^{(\gamma, \delta)} \delta_{m n}$,

where,

$$
h_{n}^{(\gamma, \delta)}=\frac{2^{\gamma+\delta+1} \Gamma(n+\gamma+1) \Gamma(n+\delta+1)}{(2 n+\gamma+\delta+1) n ! \Gamma(n+\gamma+\delta+1)},
$$

and $\delta_{m n}$ is the well-known kronecker delta function. These polynomials are eigenfunctions of the following singular Sturm-Liouville equation

$\left(1-x^{2}\right) u^{\prime \prime}+(\delta-\gamma-(\gamma+\delta+2) x) u^{\prime}+n(n+\gamma+\delta+1) u=0$.

Also, Jacobi polynomials may be generated by means of Rodrigue's formula

$P_{n}^{(\gamma, \delta)}(x)=\frac{(-1)^{n}}{2^{n} n ! w^{(\gamma, \delta)}(x)} \frac{d^{n}}{d x^{n}}\left[w^{(\gamma, \delta)}(x)\left(1-x^{2}\right)^{n}\right]$.

The polynomials, namely the ultraspherical, Legendre, first, second, third and fourth kinds Chebyshev polynomials, can be deduced as special cases of the Jacobi polynomials as shown in Table 1.
Table 1. Special cases of Jacobi polynomials.

\begin{tabular}{ll}
\hline Ultraspherical polynomials & $C_{n}^{(\lambda)}=\frac{(2 \lambda)_{n}}{\left(\lambda+\frac{1}{2}\right)_{n}} P_{n}^{\left(\lambda-\frac{1}{2}, \lambda-\frac{1}{2}\right)}(x)$ \\
Legendre polynomials & $L_{n}(x)=P_{n}^{(0,0)}(x)$ \\
Chebyshev polynomials of first kind & $T_{n}(x)=\frac{\left(\frac{1}{2}\right)_{n}}{n !} P_{n}^{\left(-\frac{1}{2},-\frac{1}{2}\right)}(x)$ \\
Chebyshev polynomials of second kind & $U_{n}(x)=\frac{\left(\frac{3}{2}\right)_{n}}{(n+1) !} P_{n}^{\left(\frac{1}{2}, \frac{1}{2}\right)}(x)$ \\
Chebyshev polynomials of third kind & $V_{n}(x)=\frac{\left(2^{n} n !\right)^{2}}{(2 n) !} P_{n}^{\left(-\frac{1}{2}, \frac{1}{2}\right)}(x)$ \\
Chebyshev polynomials of fourth kind & $W_{n}(x)=\frac{\left(2^{n} n !\right)^{2}}{(2 n) !} P_{n}^{\left(\frac{1}{2},-\frac{1}{2}\right)}(x)$
\end{tabular}

Note that in this table, the symbol $(a)_{n}$ is the Pochhammer symbol, $(a)_{n}=\frac{\Gamma(a+n)}{\Gamma(n)}$. For more details about Jacobi polynomials, one can see 32 34.

\subsection{Shifted Jacobi polynomials}

In order to use the Jacobi polynomials on the interval $[0,1]$, we define the so-called shifted Jacobi polynomials by introducing the change of variable $t=2 x-1$. Let the shifted Jacobi polynomials $P_{n}^{(\gamma, \delta)}(2 x-1)$ be denoted by $\rho_{n}^{(\gamma, \delta)}(x)$. Then $\rho_{n}^{(\gamma, \delta)}(x)$ can be obtained as follows:

$$
\rho_{n}^{(\gamma, \delta)}(x)=\frac{(-1)^{n}}{n ! x^{\delta}(1-x)^{\gamma}} \frac{d^{n}}{d x^{n}}\left[x^{\delta+n}(1-x)^{\gamma+n}\right] .
$$

The shifted Jacobi Polynomials $\rho_{n}^{(\gamma, \delta)}(x)$ has the orthogonality relation

$$
\int_{0}^{1} x^{\delta}(1-x)^{\gamma} \rho_{m}^{(\gamma, \delta)}(x) \rho_{n}^{(\gamma, \delta)}(x) d x=\frac{h_{n}^{(\gamma, \delta)}}{2^{\gamma+\delta+1}} \delta_{m n}
$$

The following special values will be of important use later

$$
\begin{array}{r}
\rho_{i}^{(\gamma, \delta)}(0)=\frac{(-1)^{i}(\delta+1)_{i}}{i !}, \quad \rho_{i}^{(\gamma, \delta)}(1)=\frac{(\gamma+1)_{i}}{i !}, \\
D^{q} \rho_{i}^{(\gamma, \delta)}(0)=\frac{(-1)^{i-q}(\delta+q+1)_{i-q}(i+\gamma+\delta+1)_{q}}{(i-q) !}, \\
D^{q} \rho_{i}^{(\gamma, \delta)}(1)=\frac{(-1)^{i-q}(\gamma+q+1)_{i-q}(i+\gamma+\delta+1)_{q}}{(i-q) !} .
\end{array}
$$

A function $f(t)$ defined over $[0,1]$ may be expanded in terms of the shifted Jacobi polynomials as

$$
f(t)=\sum_{i=0}^{\infty} c_{i} \rho_{i}^{(\gamma, \delta)}(t)
$$


where,

$$
c_{i}=\frac{2^{\gamma+\delta+1}}{h_{i}^{(\gamma, \delta)}} \int_{0}^{1} t^{\delta}(1-t)^{\gamma} f(t) \rho_{i}^{(\gamma, \delta)}(t) d t
$$

The following theorem gives the differentiation and integration formulae of $\rho_{i}^{(\gamma, \delta)}(t)$

Theorem 1. [35] The differentiation and integration of $\rho_{i}^{(\gamma, \delta)}(t)$ are given by the following formulae:

$$
\begin{aligned}
& \frac{d}{d t} \rho_{i}^{(\gamma, \delta)}(t)=(i+\gamma+\delta+1) \rho_{i-1}^{(\gamma+1, \delta+1)}(t), \\
& \int_{0}^{t} \rho_{i}^{(\gamma, \delta)}(s) d s \\
& =\frac{1}{i+\gamma+\delta}\left(\rho_{i+1}^{(\gamma-1, \delta-1)}(t)+\frac{(-1)^{i}(\delta)_{i+1}}{(i+1) !}\right) .
\end{aligned}
$$

\section{Numerical treatment for FOCP}

In this section, we are interested in the reformulation of the fractional optimal control problem. In addition, we introduce numerical solutions for the proposed FOCP.

\subsection{FOCP reformulation}

The fractional optimal control problem (FOCP) can be defined as follows. Find the optimal control $u(t)$ for a fractional dynamical system (FDS) that minimizes the performance index

$$
J(u)=\int_{0}^{1} F(t, x(t), u(t)) d t,
$$

subject to the dynamical system

$$
D_{t}^{\alpha} x(t)=G(t, x(t), u(t)), \alpha \in(n-1, n], t \in[0,1],
$$

subject to the initial condition

$$
x^{(i)}(0)=X_{i}, \quad i=0,1, \ldots, n-1,
$$

where $x(t)$ is the state variable, $t$ represents the time, $F$ and $G$ are two arbitrary functions and $X_{i}$ are real known constants. The necessary optimality conditions (see, [30]) of FOCP (13, 15) leads to

$$
\begin{aligned}
& { }_{0} D_{t}^{\alpha} x(t)=G(t, x(t), u(t)), \\
& { }_{t} D_{1}^{\alpha} \lambda(t)=\frac{\partial F}{\partial x}+\lambda \frac{\partial G}{\partial x}, \\
& \frac{\partial F}{\partial u}+\lambda \frac{\partial G}{\partial u}=0,
\end{aligned}
$$

and

$$
x^{(i)}(0)=X_{i}, \quad \lambda^{(i)}(1)=0 \quad i=0,1, \ldots, n-1 .
$$

The necessary conditions for the optimality of the FOCP considered are those given in (16. 19). These equations are similar to the EulerLagrange equations for classical optimal control problems except that the resulting differential equations contain the left and the right fractional derivatives. Moreover, the derivation of these equations is very similar to the derivation for an optimal control problem containing integral order derivatives. Determination of the optimal control for the fractional system requires solution of Eqs. (16) $-(19)$.

\subsection{Numerical algorithm for FOCP}

In this section, we are concerned with the numerical solutions of the coupled system of equations (16)-(19), by applying shifted Jacobi tau method. First, we use Eq. (18), to eliminate $u(t)$ from Eqs. (16) and (17) to obtain a system of coupled fractional order differential equations of the form:

$$
\begin{aligned}
& { }_{0} D_{t}^{\alpha} x(t)=G_{1}(t, x(t), \lambda(t)), \\
& { }_{t} D_{1}^{\alpha} \lambda(t)=F_{1}(t, x(t), \lambda(t)),
\end{aligned}
$$

subject to

$$
x^{(i)}(0)=X_{i}, \quad \lambda^{(i)}(1)=0 \quad i=0,1, \ldots, n-1 .
$$

We approximate $x(t), \lambda(t)$ as follows

$$
\begin{aligned}
& x(t) \approx \sum_{i=0}^{N-1} a_{i} \rho_{i}^{(\gamma, \delta)}(t), \\
& \lambda(t) \approx \sum_{i=0}^{M-1} b_{i} \rho_{i}^{(\gamma, \delta)}(t) .
\end{aligned}
$$

Now we can compute the residual of Eqs. (20) and (21) as follows:

$$
\begin{aligned}
& R_{1}(t)=\sum_{i=0}^{N-1} a_{i 0} D_{t}^{\alpha} \rho_{i}^{(\gamma, \delta)}(t) \\
& -G_{1}\left(t, \sum_{i=0}^{N-1} a_{i} \rho_{i}^{(\gamma, \delta)}(t), \sum_{i=0}^{M-1} b_{i} \rho_{i}^{(\gamma, \delta)}(t)\right), \\
& R_{2}(t)=\sum_{i=0}^{M-1} b_{i t} D_{1}^{\alpha} \rho_{i}^{(\gamma, \delta)}(t) \\
& -F_{1}\left(t, \sum_{i=0}^{N-1} a_{i} \rho_{i}^{(\gamma, \delta)}(t), \sum_{i=0}^{M-1} b_{i} \rho_{i}^{(\gamma, \delta)}(t)\right) .
\end{aligned}
$$


The application of the typical tau method yields

$$
\begin{gathered}
\int_{0}^{1} R_{1}(t) \rho_{i}^{(\gamma, \delta)}(t) d t=0, \\
i=0,1, \ldots, N-n-1, \\
\int_{0}^{1} R_{2}(t) \rho_{i}^{(\gamma, \delta)}(t) d t=0, \\
i=0,1, \ldots, M-n-1 .
\end{gathered}
$$

The derivative and integral formulae of shifted Jacobi polynomials in (11) and (12) enable one to reduce the integrals in (27) and (28) into a set of algebraic equations in the unknown expansion coefficients. Moreover, the use of the initial conditions along with the identities (8) and (9) yield

$$
\begin{aligned}
& \sum_{i=j}^{N-1} a_{i} \frac{(-1)^{i}(\delta+1)_{i}}{i !}=X_{j}, \\
& j=0,1, \ldots, n-1, \\
& \sum_{i=j}^{M-1} b_{i} \frac{(\gamma+1)_{i}}{i !}=0, \\
& j=0,1, \ldots, n-1 .
\end{aligned}
$$

Eqs. (27)-(30) generate a system of $(N+M)$ algebraic equations in the expansion coefficients, $a_{i}$ and $b_{i}$, which can be solved with the aid of the well-known Newton's iterative method.

\section{Numerical results and discussion}

In this section, the spectral-tau Jacobi algorithm (STJA) is employed for handling some FOCPs accompanied with some comparisons hoping to demonstrate the efficiency and applicability of the proposed algorithm.

Example 1. Consider the following minimization problem [31]:

$$
\begin{aligned}
& \min J=\frac{1}{2} \int_{0}^{1}\left(x^{2}+u^{2}-2 t^{2} x-2 t^{3} u+t^{4}+t^{6}\right) d t, \\
& { }_{0} D_{t}^{\alpha} x(t)=2\left(t^{1+\alpha} \Gamma(3-\alpha)\right)^{-1} u(t), \quad \alpha \in(0,1), \\
& x(0)=0,
\end{aligned}
$$

with the exact solution

$\left(\begin{array}{l}x(t) \\ u(t)\end{array}\right)=\left(\begin{array}{c}t^{2} \\ t^{3}\end{array}\right)$.

This solution minimizes the performance index $J$, and the minimum value is $\mu=0$. Applying the procedures explained in Section 3, we get the following coupled system:

$$
\begin{aligned}
& { }_{0} D_{t}^{\alpha} x(t)=2\left(t^{1+\alpha} \Gamma(3-\alpha)\right)^{-1} \\
& \times\left[t^{3}-2\left(t^{1+\alpha} \Gamma(3-\alpha)\right)^{-1} \lambda(t)\right], \\
& { }_{t} D_{1}^{\alpha} \lambda(t)=x(t)-t^{2}, \\
& x(0)=0, \quad \lambda(1)=1 .
\end{aligned}
$$

If we apply STJA with $N=M=4$, then we have

$$
\begin{aligned}
x(t) & \approx \sum_{i=0}^{3} a_{i} \rho_{i}^{(\gamma, \delta)}(t), \\
\lambda(t) & \approx \sum_{i=0}^{3} b_{i} \rho_{i}^{(\gamma, \delta)}(t) .
\end{aligned}
$$

Now the residuals of (31), (32) can be written in the following formulae

$$
\begin{aligned}
R_{1}(t) & =\sum_{i=0}^{3} a_{i 0} D_{t}^{\alpha} \rho_{i}^{(\gamma, \delta)}(t)- \\
2 & \left(t^{1+\alpha} \Gamma(3-\alpha)\right)^{-1} \\
& \times\left[t^{3}-2\left(t^{1+\alpha} \Gamma(3-\alpha)\right)^{-1} \sum_{i=0}^{3} b_{i} \rho_{i}^{(\gamma, \delta)}(t)\right], \\
R_{2}(t) & =\sum_{i=0}^{3} b_{i t} D_{1}^{\alpha} \rho_{i}^{(\gamma, \delta)}(t)-\sum_{i=0}^{3} a_{i} \rho_{i}^{(\gamma, \delta)}(t)+t^{2} .
\end{aligned}
$$

The application of tau method yields

$$
\begin{aligned}
& \int_{0}^{1} R_{1}(t) \rho_{j}^{(\gamma, \delta)}(t) d t=0, \quad j=0,1,2, \\
& \int_{0}^{1} R_{2}(t) \rho_{j}^{(\gamma, \delta)}(t) d t=0, \quad j=0,1,2 .
\end{aligned}
$$

Moreover, the initial conditions lead to the following two equations:

$$
\begin{aligned}
& \sum_{i=0}^{3} a_{i} \frac{(-1)^{i}(\delta+1)_{i}}{i !}=0, \\
& \sum_{i=0}^{3} b_{i} \frac{(\gamma+1)_{i}}{i !}=1,
\end{aligned}
$$

The system in (38)-(41) can be solved to give

$$
\begin{gathered}
a_{0}=\frac{(1+\delta)_{2}}{(2+\gamma+\delta)_{2}}, a_{1}=\frac{2(2+\delta)}{(2+\gamma+\delta)(4+\gamma+\delta)}, \\
a_{2}=\frac{2}{(3+\gamma+\delta)_{2}}, a_{3}=0
\end{gathered}
$$

and

$$
b_{i}=0, \quad 0 \leq i \leq 3 .
$$


Hence

$$
x(t)=t^{2}, \quad \lambda(t)=0 .
$$

Finally, and based on Eq. (18), we get

$$
u(t)=t^{3},
$$

which are the exact solutions.

Example 2. Consider the following FOCP (see, [31]):

$$
\begin{aligned}
& \min J=\frac{1}{2} \int_{0}^{1}\left[\left(x-t^{2}\right)^{2}+\left(u+t^{4}-\frac{20 t^{\frac{9}{10}}}{9 \Gamma\left(\frac{9}{10}\right)}\right)^{2}\right] \\
& { }_{0} D_{t}^{1.1} x(t)=t^{2} x(t)+u(t), \\
& x(0)=x^{\prime}(0)=0
\end{aligned}
$$

with solution

$$
\left(\begin{array}{l}
x(t) \\
u(t)
\end{array}\right)=\left(\begin{array}{c}
t^{2} \\
\frac{20 t \frac{9}{10}}{9 \Gamma\left(\frac{9}{10}\right)}-t^{4}
\end{array}\right) .
$$

This solution minimizes the performance index $J$ and the minimum value is $\mu=0$. In Table 2, we introduce the maximum absolute error

$$
E:=\max \left(\max _{t \in[0,1]}\left|x-x_{N}\right|, \max _{t \in[0,1]}\left|u-u_{M}\right|\right)
$$

by using STJA for various choices of $N, M, \gamma$ and $\delta$.

Remark 1. It is worthy to note that the best error obtained here is $3.28 \cdot 10^{-17}$ for $(N, M)=(5,5)$ and $(\gamma, \delta)=\left(-\frac{1}{2}, \frac{1}{2}\right)$, while the best error obtained in [31] is $7.03 .10^{-8}$, for $(M, N)=(8,9)$.

Example 3. Consider the following time invariant FOCP: Find the control function $u(t)$ which minimizes the performance index (see, [9]):

$$
\begin{aligned}
\min J & =\frac{1}{2} \int_{0}^{1}\left[(x(t))^{2}+(u(t))^{2}\right] d t, \\
{ }_{0} D_{t}^{\alpha} x(t) & =-x(t)+u(t), \quad 0<\alpha \leqslant 1 \\
x(0) & =1 .
\end{aligned}
$$

The exact solution for this problem in case $\alpha=1$, is

$$
\begin{gathered}
\left(\begin{array}{c}
x(t) \\
u(t)
\end{array}\right) \\
=\left(\begin{array}{c}
c_{0} \sinh (\sqrt{2} t)+\cosh (\sqrt{2} t) \\
\left(c_{0}+\sqrt{2}\right) \sinh (\sqrt{2} t)+\left(\sqrt{2} c_{0}+1\right) \cosh (\sqrt{2} t)
\end{array}\right),
\end{gathered}
$$

where

$c_{0}=-\frac{\sqrt{2} \sinh (\sqrt{2})+\cosh (\sqrt{2})}{\sinh (\sqrt{2})+\sqrt{2} \cosh (\sqrt{2})}$.
In Figure 1, we illustrate the exact solution in case of $\alpha=1$, and the STJA solution for the case corresponds to $(N, M)=(7,7),(\gamma, \delta)=\left(-\frac{1}{2}, \frac{1}{2}\right)$ (Chebyshev third kind case) and different values of $\alpha$. Figure 1 shows the state and the control variables, respectively, as a function of time. From this figure it is clear that both the state and the control variables is close to the solution in the integral value of $\alpha$ as expected.

Example 4. Consider a linear time varying system with the same performance index and the same initial condition as those considered in Exdtample 3, except in this example, the system is subjected to the following dynamic constraint:

$$
{ }_{0} D_{t}^{\alpha} x(t)=t x(t)+u(t), \quad 0<\alpha \leqslant 1,
$$

the exact solution for this problem in case $\alpha=1$, is

$$
\left(\begin{array}{l}
x(t) \\
u(t)
\end{array}\right)=\left(\begin{array}{c}
c_{1} e^{\frac{t^{2}}{2}} \sin (t)+e^{\frac{t^{2}}{2}} \cos (t) \\
c_{1} e^{\frac{t^{2}}{2}} \cos (t)-e^{\frac{t^{2}}{2}} \sin (t)
\end{array}\right),
$$

where $c_{1}=\tan (1)$. In Figure 2, we illustrate the exact solution in case of $\alpha=1$, and the STJA solution for the case corresponds to $(N, M)=(8,8)$, $(\gamma, \delta)=\left(\frac{1}{2},-\frac{1}{2}\right)$ (Chebyshev fourth kind case) and different values of $\alpha$. Figure 2 shows the state and the control variables, respectively, as a function of time. From this figure it is clear that both the state and the control variables is close to the solution in the integral value of $\alpha$ as expected.

Example 5. Consider the following nonlinear FOCP (see, [36]):

$$
\begin{aligned}
& \min \quad J=\int_{0}^{1}\left[e^{t^{3 / 2}-t} u(t)-2 t^{3 / 2} x(t)\right. \\
& +\frac{1}{4} e^{2 t^{3 / 2}-2 t}-\frac{3}{8} \sqrt{\pi} e^{t^{3 / 2}-2 t} \\
& +t^{3}+u(t)^{2}-\frac{3}{4} \sqrt{\pi} e^{-t} u(t)+x(t)^{2} \\
& \left.+e^{2 t}+\frac{9}{64} \pi e^{-2 t}\right] d t \\
& { }_{0} D_{t}^{1.5} x(t)=e^{x(t)}+2 e^{t} u(t), \\
& x(0)=x^{\prime}(0)=0 .
\end{aligned}
$$

The exact solution for this problem is $\left(\begin{array}{l}x(t) \\ u(t)\end{array}\right)=\left(\begin{array}{c}t^{\frac{3}{2}} \\ \frac{1}{2} e^{-t}\left(\frac{3 \sqrt{\pi}}{4}-e^{t^{\frac{3}{2}}}\right)\end{array}\right)$, which minimizes the performance index $J$ and the minimum value is $\frac{1}{2}\left(e^{2}-1\right) \approx$ 3.1945280494653251. In Table 3, we introduce the maximum absolute error 
Table 2. Maximum absolute error of Example 2,

\begin{tabular}{ccccccc}
\hline$(N, M)$ & $(\gamma, \delta)$ & $E$ & $(\gamma, \delta)$ & $E$ & $(\gamma, \delta)$ & $E$ \\
\hline$(3,4)$ & & $8.40 \cdot 10^{-10}$ & & $5.87 \cdot 10^{-10}$ & $2.32 \cdot 10^{-10}$ \\
$(4,3)$ & $(0,0)$ & $6.85 \cdot 10^{-10}$ & $\left(-\frac{1}{2},-\frac{1}{2}\right)$ & $1.02 \cdot 10^{-10}$ & $\left(\frac{1}{2}, \frac{1}{2}\right)$ & $1.85 \cdot 10^{-10}$ \\
$(5,5)$ & & $9.31 \cdot 10^{-16}$ & & $5.27 \cdot 10^{-16}$ & & $3.25 \cdot 10^{-16}$ \\
\hline$(3,4)$ & & $9.24 \cdot 10^{-10}$ & & $6.38 \cdot 10^{-10}$ & & $2.32 \cdot 10^{-10}$ \\
$(4,3)$ & $\left(-\frac{1}{2}, \frac{1}{2}\right)$ & $4.28 \cdot 10^{-10}$ & $\left(-\frac{1}{2}, \frac{1}{2}\right)$ & $1.02 \cdot 10^{-10}$ & $(1,2)$ & $1.85 \cdot 10^{-10}$ \\
$(5,5)$ & & $3.28 \cdot 10^{-17}$ & & $8.27 \cdot 10^{-17}$ & & $5.75 \cdot 10^{-16}$ \\
\hline
\end{tabular}

$$
E:=\max \left(\max _{t \in[0,1]}\left|x-x_{N}\right|, \max _{t \in[0,1]}\left|u-u_{M}\right|\right)
$$

by using STJA for various choices of $N, M, \gamma$ and $\delta$.

Remark 2. It is worthy noting here, for $M=N=16$ and $(\gamma, \delta)=\left(\frac{1}{2}, \frac{1}{2}\right)$ and $\left(-\frac{1}{2}, \frac{1}{2}\right)$, we get $J=3.1945280494653253$ and 3.1945280494653258 which is almost the exact value.

\section{Concluding Remarks}

In this paper, we presented an efficient numerical algorithm for some spectral solutions of a class of fractional optimal control problems. The proposed numerical solutions are expressed as expansions of Jacobi polynomials. The celebrated spectral tau method is utilized for this purpose. The numerical tests clarify that our method is applicable and it is more accurate than some other methods in literature.

\section{Acknowledgments}

The authors would like to thank the referees and the editor for their constructive comments; which helped substantially to improve the manuscript.

\section{References}

[1] Brunner, H., Pedas, A. and Vainikko, V. (2001). Piecewise polynomial collocation methods for linear Volterra integro-differential equations with weakly singular kernels. SIAM J. Numer. Anal., 39(3),957982.

[2] Kilbas, A.A., Trujillo, J.J. and Srivastava, H.M. (2006). Theory and applications of fractional differential equations, volume 204. Elsevier Science Limited.

[3] Podlubny, I. (1998). Fractional Differential Equations: An Introduction to Fractional Derivatives, Fractional Differential Equations, to Methods of Their Solution and Some of Their Applications, volume 198. Academic press.

[4] Stefan, S.G., Kilbas, A.A. and Marichev, O.T. (1993). Fractional integrals and derivatives. Theory and $A p$ plications, Gordon and Breach, Yverdon.
[5] Al-Mdallal, Q.M., Syam, M.I. and Anwar, M.N. (2010). A collocation-shooting method for solving fractional boundary value problems. Commun. Nonlinear Sci. Numer. Simul., 15(12), 3814-3822.

[6] Çenesiz, Y., Keskin, Y. and Kurnaz, A. (2010). The solution of the Bagley-Torvik equation with the generalized Taylor collocation method. J. Franklin Inst., 347(2), 452-466.

[7] Daftardar-Gejji, V. and Jafari, H. (2005). Adomian decomposition: a tool for solving a system of fractional differential equations. J. Math. Anal. Appl., 301(2), 508-518.

[8] Momani, S. (2007). An algorithm for solving the fractional convection-diffusion equation with nonlinear source term. Commun. Nonlinear Sci. Numer. Simul., 12(7), 1283-1290.

[9] Agrawal, O.P. (2004). A general formulation and solution scheme for fractional optimal control problems. Nonlinear Dynam., 38(1-4), 323-337.

[10] Erturk, V.S., Momani, S. and Odibat, Z. (2008). Application of generalized differential transform method to multi-order fractional differential equations. Commun. Nonlinear Sci. Numer. Simul., 13(8), 1642-1654.

[11] Agrawal, O.P. and Baleanu, D. (2007). A Hamiltonian formulation and a direct numerical scheme for fractional optimal control problems. J. Vib. Control, 13(910), 1269-1281.

[12] Abd-Elhameed, W.M. and Youssri, Y.H. (2015). New spectral solutions of multi-term fractional order initial value problems with error analysis. CMES:Comp. Model. Eng. Sci, 105, 375-398.

[13] Baleanu, D., Ozlem, D., and Agrawal, O.P. (2009). A central difference numerical scheme for fractional optimal control problems. Journal of Vibration and Control, 15(4), 583-597.

[14] Agrawal, O.P., Ozlem, D. and Baleanu, D. (2010). Fractional optimal control problems with several state and control variables. Journal of Vibration and Control, 16(13), 1967-1976.

[15] Shen, J., Tang, T. and Wang, L-L. (2011). Spectral Methods: Algorithms, Analysis and Applications, volume 41. Springer Science \& Business Media.

[16] Kopriva, D.A. (2009). Implementing spectral methods for partial differential equations: algorithms for scientists and engineers. Springer Science \& Business Media.

[17] Shizgal, S. (2015) Spectral methods in chemistry and physics. Springer.

[18] Doha, E.H., Abd-Elhameed, W.M. and Bhrawy, A.H. (2013). New spectral-Galerkin algorithms for direct solution of high even-order differential equations using symmetric generalized Jacobi polynomials. Collect. Math., 64(3), 373-394. 
Table 3. Maximum absolute error of Example 5]

\begin{tabular}{ccccccc}
\hline$N=M$ & $(\gamma, \delta)$ & $E$ & $(\gamma, \delta)$ & $E$ & $(\gamma, \delta)$ & $E$ \\
\hline 4 & & $2.35 \cdot 10^{-4}$ & & $8.24 \cdot 10^{-4}$ & & $2.22 \cdot 10^{-4}$ \\
10 & $(0,0)$ & $5.91 \cdot 10^{-10}$ & $\left(-\frac{1}{2},-\frac{1}{2}\right)$ & $5.27 \cdot 10^{-10}$ & $\left(\frac{1}{2}, \frac{1}{2}\right)$ & $5.86 \cdot 10^{-10}$ \\
16 & & $3.29 \cdot 10^{-16}$ & & $8.61 \cdot 10^{-16}$ & & $1.11 \cdot 10^{-16}$ \\
\hline 4 & & $4.19 \cdot 10^{-4}$ & & $3.61 \cdot 10^{-4}$ & & $2.54 \cdot 10^{-4}$ \\
10 & $\left(-\frac{1}{2}, \frac{1}{2}\right)$ & $7.72 \cdot 10^{-10}$ & $\left(-\frac{1}{2}, \frac{1}{2}\right)$ & $2.58 \cdot 10^{-10}$ & $(1,2)$ & $6.41 \cdot 10^{-10}$ \\
16 & & 2.22.10 $10^{-17}$ & & 3.12.10 $10^{-17}$ & & $1.11 \cdot 10^{-16}$ \\
\hline
\end{tabular}
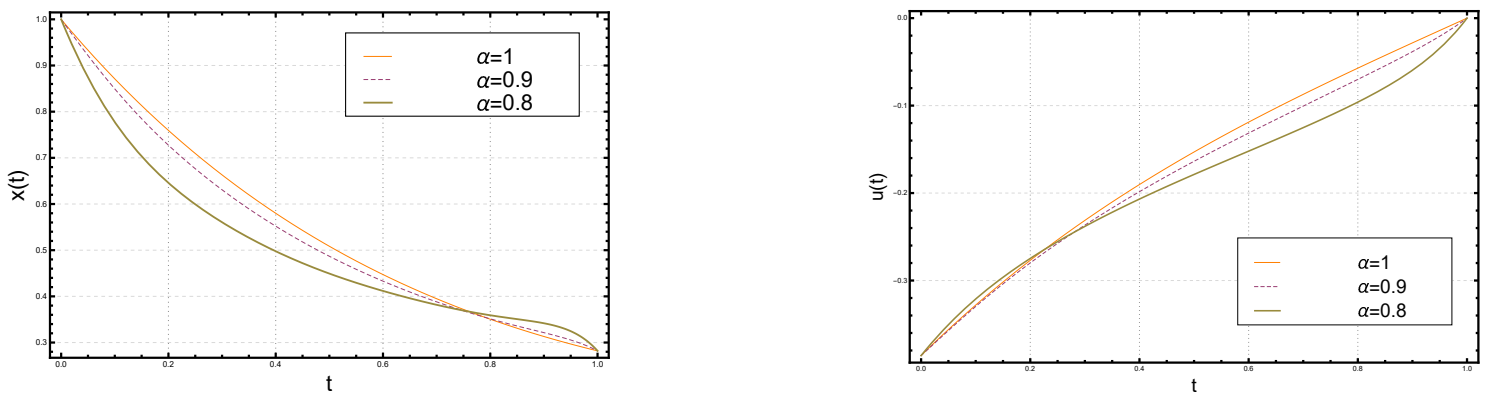

Figure 1. Different solutions of Example 3
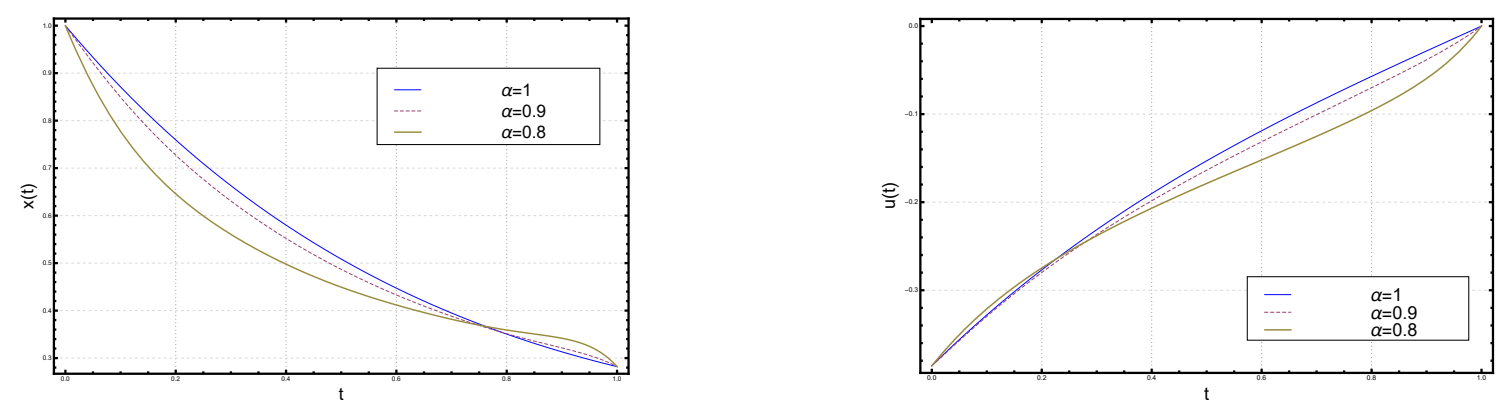

Figure 2. Different solutions of Example 4.

[19] Doha, E.H., Abd-Elhameed, W.M. and Bassuony, M.A. (2013). New algorithms for solving high evenorder differential equations using third and fourth Chebyshev-Galerkin methods. J. Comput. Phys., 236, 563-579.

[20] Abd-Elhameed, W.M., Doha, E.H. and Youssri, Y.H. (2013). New wavelets collocation method for solving second-order multipoint boundary value problems using Chebyshev polynomials of third and fourth kinds. Abstr. Appl. Anal., Volume 2013:Article ID 542839, 9 pages.

[21] Abd-Elhameed, W.M., Doha, E.H., and Youssri, Y.H. (2013). New spectral second kind chebyshev wavelets algorithm for solving linear and nonlinear second-order differential equations involving singular and bratu type equations. Abstr. Appl. Anal., Volume2013:Article ID 715756, 9 pages.

[22] Doha, E.H., Abd-Elhameed, W.M. and Youssri, Y.H. (2013). Second kind Chebyshev operational matrix algorithm for solving differential equations of LaneEmden type. New Astro., 23, 113-117.

[23] Abd-Elhameed, W.M. (2015). New Galerkin operational matrix of derivatives for solving Lane-Emden singular-type equations. Eur. Phys. J. Plus, 130(3), 52 .
[24] Abd-Elhameed, W.M., Doha, E.H., and Youssri, Y.H. (2013). Efficient spectral-Petrov-Galerkin methods for third-and fifth-order differential equations using general parameters generalized jacobi polynomials. Quaest. Math., 36(1), 15-38.

[25] Doha, E.H. and Abd-Elhameed, W.M. (2014). On the coefficients of integrated expansions and integrals of Chebyshev polynomials of third and fourth kinds. Bull. Malays. Math. Sci. Soc., 37(2), 383-398.

[26] Bhrawy, A.H., and Zaky, M.A. (2016). Shifted fractional-order Jacobi orthogonal functions: application to a system of fractional differential equations. Applied Mathematical Modelling, 40(2), 832-845.

[27] Zaky, M.A., and Machado, J.A.T. (2017). On the formulation and numerical simulation of distributedorder fractional optimal control problems. Communications in Nonlinear Science and Numerical Simulation, 52, 177-189.

[28] Bhrawy, A.H., Zaky, M.A. and Machado, J.A.T. (2017). Numerical solution of the two-sided spacetime fractional telegraph equation via Chebyshev Tau approximation. Journal of Optimization Theory and Applications, 174(1), 321-341. 
[29] Zaky, M.A. (2017). A Legendre spectral quadrature tau method for the multi-term time-fractional diffusion equations. Computational and Applied Mathematics, 1-14.

[30] Agrawal, O.P. (2008). A formulation and numerical scheme for fractional optimal control problems. J. Vib. Control, 14(9-10), 1291-1299.

[31] Lotfi, A., Yousefi, S.A. and Dehghan, M. (2013). Numerical solution of a class of fractional optimal control problems via the Legendre orthonormal basis combined with the operational matrix and the Gauss quadrature rule. J. Comput. Appl. Math., 250, 143160.

[32] Abramowitz, M. and Stegun, I.A. editors. (1970). Handbook of Mathematical Functions (applied mathematics Series 55). National Bureau of Standards, New York.

[33] Andrews, G.E., Askey, R. and Roy, R. (1999) Special functions. Cambridge University Press, Cambridge.

[34] Szego, G. (1939). Orthogonal polynomials, volume 23. American Mathematical Soc.

[35] Rainville, E.D. (1960). Special functions. The Macmillan Company, New York.
[36] Keshavarz, E., Ordokhani, Y. and Razzaghi, M. (2015). A numerical solution for fractional optimal control problems via bernoulli polynomials. Journal of Vibration and Control, 22(18), 3889-3903.

Youssri H. Youssri is Tenured assistant professor at Cairo University, he received a master's (2011) and doctorate (2014) in mathematics from Cairo University, Egypt. He has published more than 30 papers in different areas of Mathematics, including, orthogonal polynomials, spectral solutions of ordinary and fractional differential equations, boundary-value problems, initial-value problems and wavelets.

Waleed M. Abd-Elhameed is full professor of mathematics at Cairo University, he is a brilliant scientist in the field of spectral solution of differential equations and orthogonal polynomials. He has published more than 50 papers in different areas of Pure Mathemat$i c s$.

An International Journal of Optimization and Control: Theories \& Applications (http://ijocta.balikesir.edu.tr)

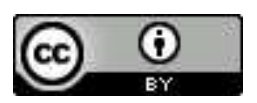

This work is licensed under a Creative Commons Attribution 4.0 International License. The authors retain ownership of the copyright for their article, but they allow anyone to download, reuse, reprint, modify, distribute, and/or copy articles in IJOCTA, so long as the original authors and source are credited. To see the complete license contents, please visit http://creativecommons.org/licenses/by/4.0/. 